\title{
Nonparaxial Propagation of Vectorial Elliptical Gaussian Beams
}

\author{
Wang Xun, Huang Kelin, Liu Zhirong, and Zhao Kangyi \\ Department of Applied Physics, East China Jiaotong University, Nanchang, Jiangxi 330013, China
}

Correspondence should be addressed to Liu Zhirong; liuzhirong_2003@126.com

Received 6 October 2015; Accepted 23 November 2015

Academic Editor: Roberto Morandotti

Copyright (C) 2016 Wang Xun et al. This is an open access article distributed under the Creative Commons Attribution License, which permits unrestricted use, distribution, and reproduction in any medium, provided the original work is properly cited.

\begin{abstract}
Based on the vectorial Rayleigh-Sommerfeld diffraction integral formulae, analytical expressions for a vectorial elliptical Gaussian beam's nonparaxial propagating in free space are derived and used to investigate target beam's propagation properties. As a special case of nonparaxial propagation, the target beam's paraxial propagation has also been examined. The relationship of vectorial elliptical Gaussian beam's intensity distribution and nonparaxial effect with elliptic coefficient $\alpha$ and waist width related parameter $f_{\omega}$ has been analyzed. Results show that no matter what value of elliptic coefficient $\alpha$ is, when parameter $f_{\omega}$ is large, nonparaxial conclusions of elliptical Gaussian beam should be adopted; while parameter $f_{\omega}$ is small, the paraxial approximation of elliptical Gaussian beam is effective. In addition, the peak intensity value of elliptical Gaussian beam decreases with increasing the propagation distance whether parameter $f_{\omega}$ is large or small, and the larger the elliptic coefficient $\alpha$ is, the faster the peak intensity value decreases. These characteristics of vectorial elliptical Gaussian beam might find applications in modern optics.
\end{abstract}

\section{Introduction}

With the development of laser technology, research on semiconductor lasers [1], microoptical technologies [2-4], and highly focusing field [5-7] has become deeper. In practical application, the problem that would be confronted is of a beam with large divergence angle or small spot size that is of the order of light wavelength. In this case, the theory of optical propagation and transformation based on paraxial approximation is no longer valid [8], and it needs strict electromagnetic field theory to solve the problem of beam's nonparaxial propagation. In recent decades, several research methods about solving beam's nonparaxial propagation have been developed, such as vectorial Rayleigh-Sommerfeld diffraction integral method [9], perturbation power series method [10], transition operators [11], angular spectrum representation [12], and virtual source point technique [13]. And vectorial Rayleigh-Sommerfeld diffraction method has been used to treat various beam's nonparaxial propagation problems [14-17].

An elliptical Gaussian beam can be radiated and realized by semiconductor diode laser [18]. In the past few years, some nonparaxial propagation properties of vectorial elliptical Gaussian beams have been reported, such as the far-field beam divergence angle [19], diffracted at a circular and a rectangular aperture $[20,21]$. Since the semiconductor laser beam has a large divergence angle, it would become necessary to consider the target beam's nonparaxial propagation. In this work, we use the vectorial Rayleigh-Sommerfeld diffraction integral formulae to solve the nonparaxial propagation of a vectorial elliptical Gaussian beam. Target beam's nonparaxial propagation analytical expressions are derived and used to investigate its propagation properties, including the evolution of intensity and shape of elliptical Gaussian beam with different elliptic coefficient $\alpha$ and different waist width related parameter $f_{\omega}$, and the relationships of elliptical Gaussian beam's nonparaxial effect and its intensity distributions with elliptic coefficient $\alpha$ as well as parameter $f_{\omega}$ are analyzed.

\section{Nonparaxial Propagation of Vectorial Elliptical Gaussian Beams in Free Space}

Let us consider the incident field of elliptical Gaussian beam, which is polarized in the $x$ direction and can be defined by

$$
\begin{aligned}
E_{x}\left(\mathbf{r}_{0}, 0\right) & =\left\{\begin{array}{l}
E_{x}\left(x_{0}, y_{0}, 0\right) \\
E_{y}\left(\mathbf{r}_{0}, 0\right)
\end{array}\right. \\
& =\left\{\begin{array}{l}
E_{0} \exp \left[-\frac{x_{0}^{2}+\left(\alpha y_{0}\right)^{2}}{\omega^{2}}\right] \\
0,
\end{array}\right.
\end{aligned}
$$


where $\mathbf{r}_{0}=x_{0} \mathbf{i}+y_{0} \mathbf{j}$ and $\mathbf{i}$ and $\mathbf{j}$ are the unit vectors in $x$ and $y$ directions, respectively. $E_{0}$ is a constant, $\omega$ is the waist width, and $\alpha$ is elliptic coefficient, which denotes the ratio of elliptical Gaussian beam's waist width in $x$ and $y$ directions.

According to the vectorial Rayleigh-Sommerfeld diffraction integral formulae, the nonparaxial propagation of light beam in the half-space $z>0$ turns out to be [9]

$$
\begin{aligned}
& E_{x}(x, y, z)=-\frac{1}{2 \pi} \iint_{-\infty}^{\infty} E_{x}\left(x_{0}, y_{0},\right. \\
& 0) \frac{\partial \mathbf{R}\left(\mathbf{r}, \mathbf{r}_{0}\right)}{\partial z} d x_{0} d y_{0}, \\
& E_{y}(x, y, z)=-\frac{1}{2 \pi} \iint_{-\infty}^{\infty} E_{y}\left(x_{0}, y_{0},\right. \\
& 0) \frac{\partial \mathbf{R}\left(\mathbf{r}, \mathbf{r}_{0}\right)}{\partial z} d x_{0} d y_{0}, \\
& E_{z}(x, y, z)=\frac{1}{2 \pi} \iint_{-\infty}^{\infty}\left(E_{x}\left(x_{0}, y_{0}, 0\right) \frac{\partial \mathbf{R}\left(\mathbf{r}, \mathbf{r}_{0}\right)}{\partial x}\right. \\
& +E_{y}\left(x_{0}, y_{0},\right. \\
& 0)) d x_{0} d y_{0} \frac{\partial \mathbf{R}\left(\mathbf{r}, \mathbf{r}_{0}\right)}{\partial y},
\end{aligned}
$$

where $\mathbf{r}=x \mathbf{i}+y \mathbf{j}+z \mathbf{k}$ and $\mathbf{k}$ denotes the unit vector in $z$ direction. $E_{x, y, z}(x, y, z)$ are components of the $E$ vector along $x, y$, and $z$ directions in an arbitrary plane $z$, respectively,

$$
\mathbf{R}\left(\mathbf{r}, \mathbf{r}_{0}\right)=\frac{\exp \left(i k\left|\mathbf{r}-\mathbf{r}_{0}\right|\right)}{\left|\mathbf{r}-\mathbf{r}_{0}\right|}
$$

where $k=2 \pi / \lambda$ is the wave number and $\lambda$ is the incident wavelength. When $\left|\mathbf{r}-\mathbf{r}_{0}\right| \gg \lambda,\left|\mathbf{r}-\mathbf{r}_{0}\right|$ can be approximately expanded into [19]

$$
\left|\mathbf{r}-\mathbf{r}_{0}\right| \approx r+\frac{x_{0}^{2}+y_{0}^{2}-2 x x_{0}-2 y y_{0}}{2 r} .
$$

So (3) can be expressed as

$$
\mathbf{R}\left(\mathbf{r}, \mathbf{r}_{0}\right)=\frac{1}{r} \exp \left[i k\left(r+\frac{x_{0}^{2}+y_{0}^{2}-2 x x_{0}-2 y y_{0}}{2 r}\right)\right],
$$

where $r=\left(x^{2}+y^{2}+z^{2}\right)^{1 / 2}$.

Substituting (5) into (2a)-(2c), we obtain

$$
\begin{aligned}
& E_{x}(x, y, z)=-\frac{i z}{\lambda r^{2}} \iint_{-\infty}^{\infty} E_{x}\left(x_{0}, y_{0}, 0\right) \\
& \cdot \exp \left[i k\left(r+\frac{r_{0}^{2}-2 x x_{0}-2 y_{0} y}{2 r}\right)\right] d x_{0} d y_{0}, \\
& E_{y}(x, y, z)=-\frac{i z}{\lambda r^{2}} \iint_{-\infty}^{\infty} E_{y}\left(x_{0}, y_{0}, 0\right) \\
& \cdot \exp \left[i k\left(r+\frac{r_{0}^{2}-2 x x_{0}-2 y_{0} y}{2 r}\right)\right] d x_{0} d y_{0},
\end{aligned}
$$

$$
\begin{aligned}
& E_{z}(x, y, z)=\frac{i}{\lambda r^{2}} \iint_{-\infty}^{\infty}\left[\left(x-x_{0}\right) E_{x}\left(x_{0}, y_{0}, 0\right)\right. \\
& \left.+\left(y-y_{0}\right) E_{y}\left(x_{0}, y_{0}, 0\right)\right] \\
& \quad \cdot \exp \left[i k\left(r+\frac{r_{0}^{2}-2 x x_{0}-2 y_{0} y}{2 r}\right)\right] d x_{0} d y_{0} .
\end{aligned}
$$

Substituting (1) into (6a), we can obtain

$$
\begin{gathered}
E_{x}(x, y, z)=-\frac{i z E_{0}}{\lambda r^{2}} \exp (i k r) \\
\cdot \int_{-\infty}^{\infty} \exp \left(-\frac{x_{0}^{2}}{\omega_{0}^{2}}+\frac{i k x_{0}^{2}}{2 r}-\frac{i k x x_{0}}{r}\right) d x_{0} \\
\cdot \int_{-\infty}^{\infty} \exp \left(-\frac{\alpha^{2} y_{0}^{2}}{\omega_{0}^{2}}+\frac{i k y_{0}^{2}}{2 r}-\frac{i k y y_{0}}{r}\right) d y_{0} .
\end{gathered}
$$

By utilizing the following integral formula [20]

$$
\begin{aligned}
& \int_{-\infty}^{\infty} x^{n} \exp \left(-\mu x^{2}+2 v x\right) d x \\
& =n ! \sqrt{\frac{\pi}{\mu}\left(\frac{v}{\mu}\right)^{n} \exp \left(\frac{v^{2}}{\mu}\right) \sum_{s=0}^{[n / 2]} \frac{1}{(n-2 s) ! s !}\left(\frac{\mu}{4 v^{2}}\right)^{s},}
\end{aligned}
$$

$[\operatorname{Re} \mu>0]$,

(7) can be expressed as follows:

$$
\begin{aligned}
& E_{x}(x, y, z) \\
& \quad=\frac{i E_{0} \pi z}{\lambda r^{2} \sqrt{p q}} \exp (i k r) \exp \left[\frac{k^{2}}{4 r^{2}}\left(\frac{x^{2}}{p}+\frac{y^{2}}{q}\right)\right],
\end{aligned}
$$

with $p$ and $q$ being given by

$$
\begin{aligned}
p & =k^{2} f_{\omega}^{2}-\frac{i k}{2 r}, \\
q & =\alpha^{2} k^{2} f_{\omega}^{2}-\frac{i k}{2 r}, \\
f_{\omega} & =\frac{1}{k \omega} .
\end{aligned}
$$

Similarly, substituting (1) into (6b) and (6c), and recalling integral formula (8), we can obtain other elements of the elliptical Gaussian beam:

$$
\begin{aligned}
& E_{y}(x, y, z)=0, \\
& E_{z}(x, y, z) \\
& =\frac{i E_{0} \pi x}{\lambda r^{2} \sqrt{p q}} \\
& \quad \cdot\left(1+\frac{i k}{2 p r}\right) \exp (i k r) \exp \left[-\frac{k^{2}}{4 r^{2}}\left(\frac{x^{2}}{p}+\frac{y^{2}}{q}\right)\right] .
\end{aligned}
$$




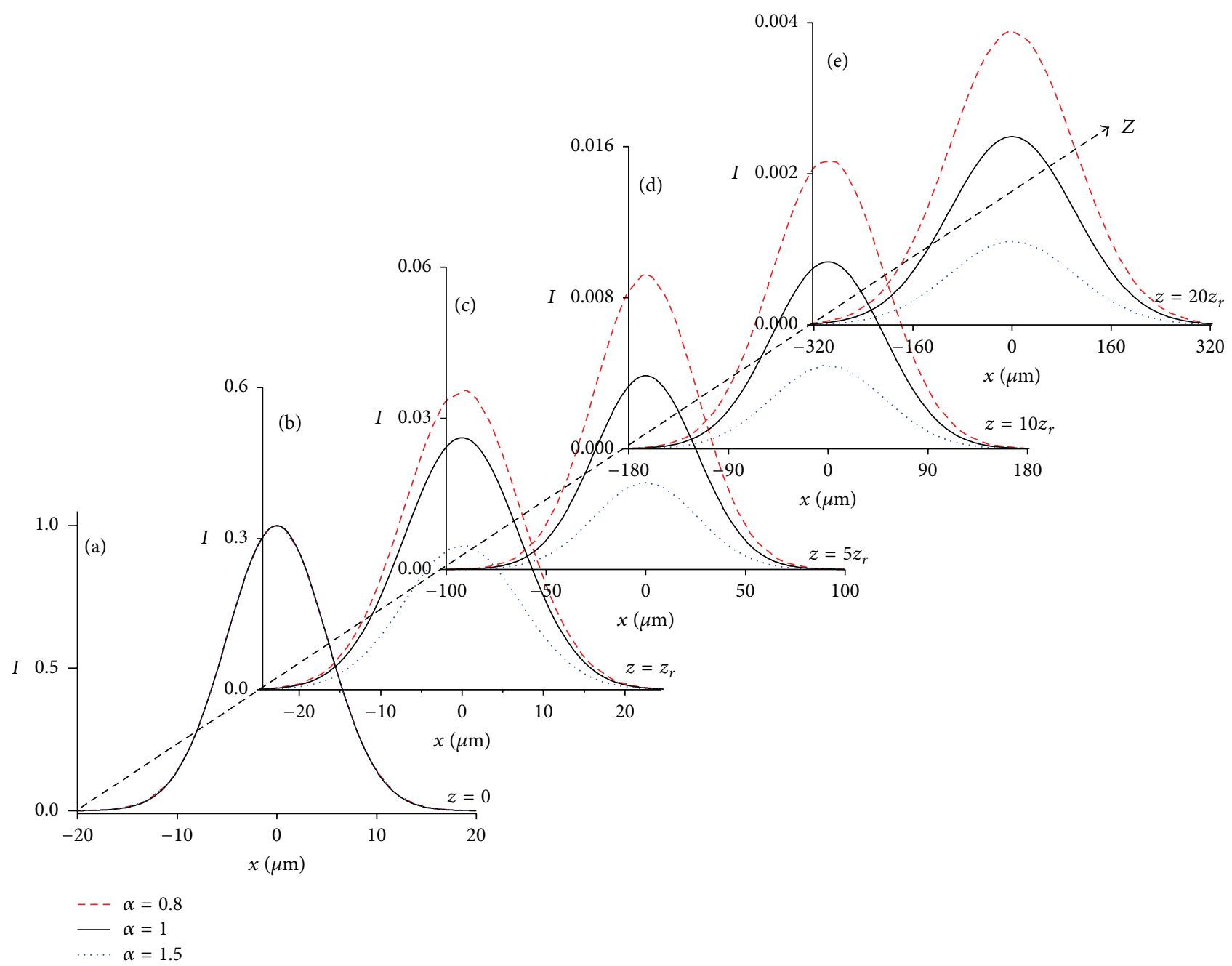

FIGURE 1: Intensity distribution of elliptical Gaussian beam for $f_{\omega}=0.01$ as a function of $x$ in free space in different planes: (a) $z=0$; (b) $z=z_{r}$; (c) $z=5 z_{r}$; (d) $z=10 z_{r}$; (e) $z=20 z_{r}$. Several curves correspond to different elliptic coefficient $\alpha: \alpha=0.8$ (dashed curve); $\alpha=1$ (solid curve); $\alpha=1.5$ (dotted curve).

The intensity distribution of nonparaxial propagation of the elliptical Gaussian beam at the point $(x, y, z)$ can be expressed as follows:

$$
\begin{aligned}
I(x, y, z)= & I_{x}(x, y, z)+I_{y}(x, y, z)+I_{z}(x, y, z) \\
= & \left|E_{x}(x, y, z)\right|^{2}+\left|E_{y}(x, y, z)\right|^{2} \\
& +\left|E_{z}(x, y, z)\right|^{2},
\end{aligned}
$$

where $I_{x}(x, y, z), I_{y}(x, y, z)$, and $I_{z}(x, y, z)$ are the intensity distributions of the $x, y$, and $z$ components of the field, respectively.

The paraxial propagation of elliptical Gaussian beam can be dealt with as a special case by using the paraxial expansion

$$
r \approx z+\frac{x^{2}+y^{2}}{2 z} .
$$

Accordingly, (7) can be reduced to

$$
\begin{aligned}
E_{p}(x, y, z)= & \frac{i E_{0} \pi}{\lambda z \sqrt{p^{\prime} q^{\prime}}} \exp (i k z) \exp \left(i k \frac{x^{2}+y^{2}}{2 z}\right) \\
& \cdot \exp \left[\frac{k^{2}}{4 z^{2}}\left(\frac{x^{2}}{p^{\prime}}+\frac{y^{2}}{q^{\prime}}\right)\right],
\end{aligned}
$$

where

$$
\begin{aligned}
& p^{\prime}=k^{2} f_{\omega}^{2}-\frac{i k}{2 z}, \\
& q^{\prime}=\alpha^{2} k^{2} f_{\omega}^{2}-\frac{i k}{2 z} .
\end{aligned}
$$

Equations (9) and (12) are the main analytical results for elliptical Gaussian beam's nonparaxial propagating in free space, and (15) is the paraxial analytical formula for elliptical Gaussian beam's paraxial propagating in free space. 


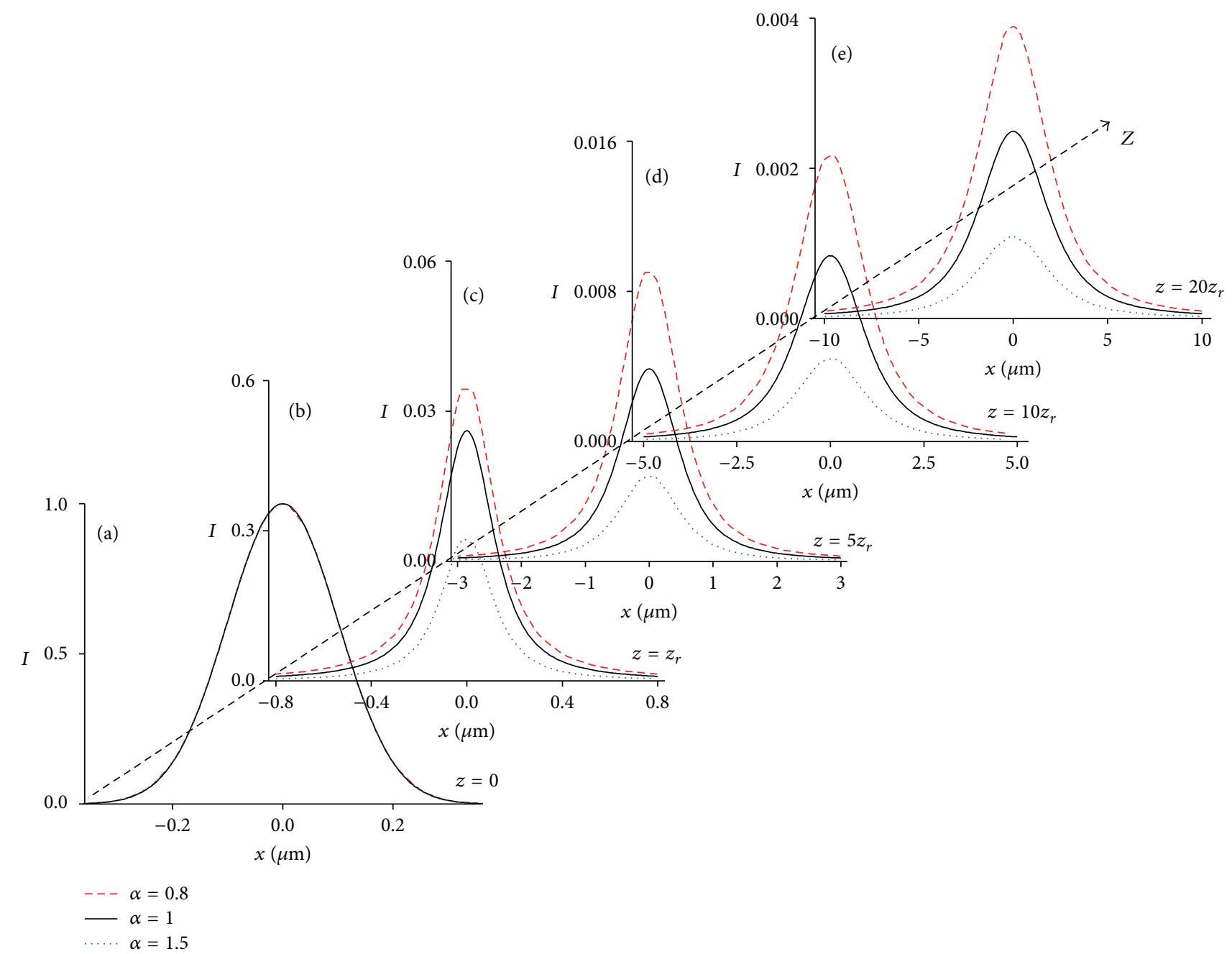

FIGURE 2: Intensity distribution of elliptical Gaussian beam for $f_{\omega}=0.5$ as a function of $x$ in free space in different planes: (a) $z=0$; (b) $z=z_{r}$; (c) $z=5 z_{r} ;$ (d) $z=10 z_{r}$; (e) $z=20 z_{r}$. Several curves correspond to different elliptic coefficient $\alpha: \alpha=0.8$ (dashed curve); $\alpha=1$ (solid curve); $\alpha=1.5$ (dotted curve).

\section{Numerical Simulations and Analysis}

In order to confirm the relationship of elliptical Gaussian beam's intensity distribution and nonparaxial effects with elliptic coefficient $\alpha$ as well as parameter $f_{\omega}$, according to the analytical expressions obtained above, we have carried out the numerical simulations of intensity distributions of vectorial elliptical Gaussian beam's nonparaxial propagating in free space. For the convenience of comparison, the light peak intensity in the input plane $z=0$ is set to 1 . The propagation distance is normalized to $z / z_{r}$, where $z_{r}=\pi \omega^{2} / \lambda$ is the Rayleigh distance, and the incident wavelength is $632.8 \mathrm{~nm}$.

The evolution behavior of intensity distributions of nonparaxial elliptical Gaussian beams with several elliptic coefficients $\alpha$ in different observation planes is depicted in Figures 1 and 2, which correspond to two different waist width related parameters $f_{\omega}$, respectively. From Figure 1, for small value $f_{\omega}=0.01$-that is, elliptical Gaussian beam's waist width $\omega$ is large-one can see that all the normalized intensity distributions of nonparaxial elliptical Gaussian beams would preserve Gaussian type when the propagation distance ranges from $z=0$ to $z=20 z_{r}$, while for large value $f_{\omega}=$ 0.5 -that is, elliptical Gaussian beam's waist width $\omega$ is small (see Figure 2)-we can find that, with the increase of propagation distance $z$, the transverse intensity profiles turn into Gaussian-like shape quickly. Besides, numerical results also show that the peak intensity value decreases when the propagation distance increases, and the larger the value of elliptic coefficient $\alpha$ is, the faster the peak intensity value decreases, no matter whether $f_{\omega}$ is large or small.

Figure 3 gives the intensity distributions of elliptical Gaussian beam in the plane $z=10 z_{r}$ for different parameter $f_{\omega}$. The elliptic coefficient $\alpha$ is fixed to $0.8,1$, and 1.5 from the first row to the third row, respectively. The corresponding longitudinal component $I_{z}$ of nonparaxial elliptical Gaussian beam and paraxial result $I_{p}$ of elliptical Gaussian beam are also depicted together for comparison. From Figures 3(a1)3 (a3), one can see that no matter what value of the elliptic coefficient $\alpha$ is, for small value of $f_{\omega}=0.1, I_{z}$ is very small and can be neglected; hence, the curves of total intensity 


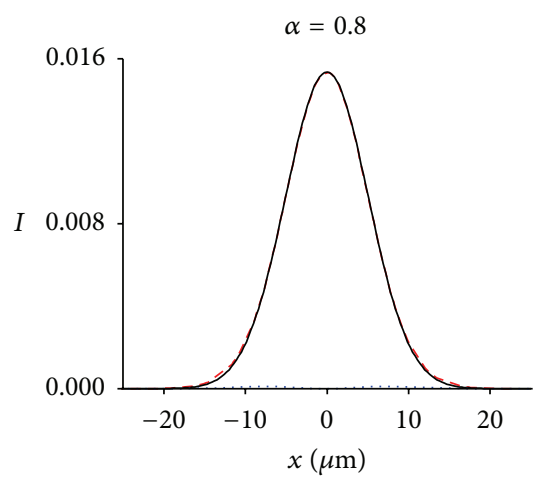

(a1)

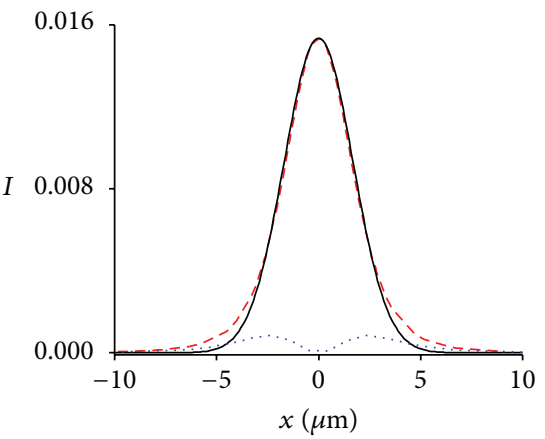

(b1)

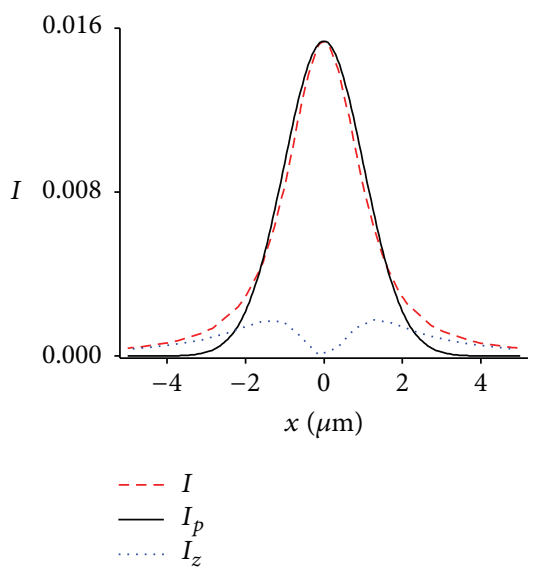

(c1)

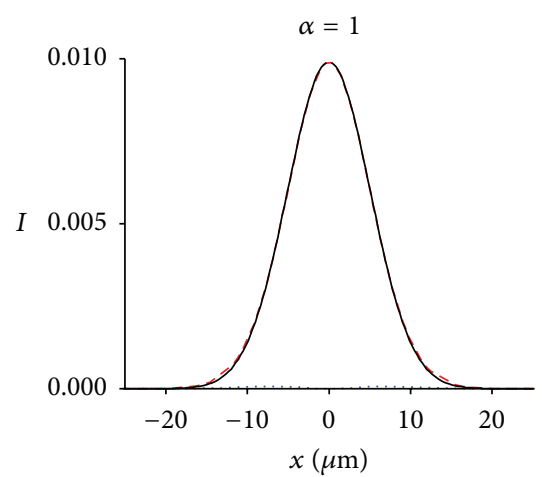

(a2)

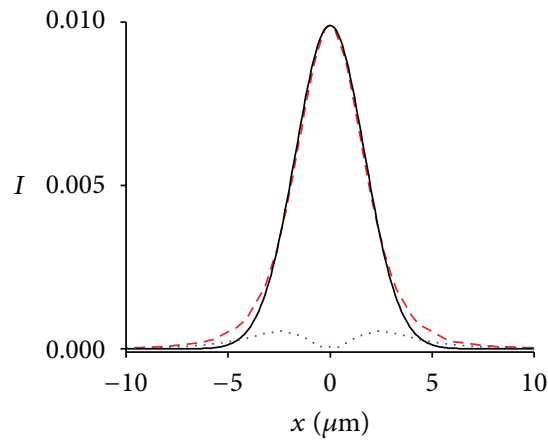

(b2)

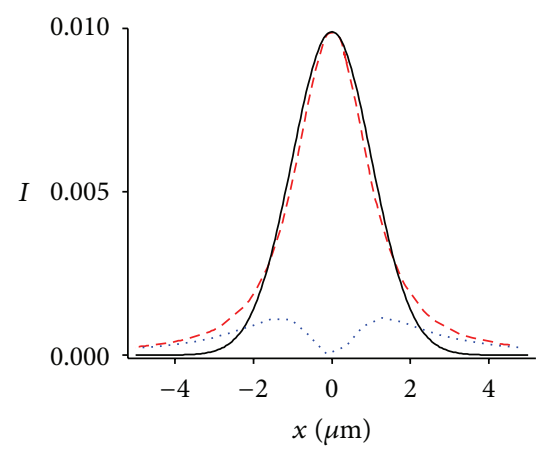

$$
\begin{array}{ll}
--- & I \\
- & I_{p} \\
\ldots \ldots & I_{z}
\end{array}
$$

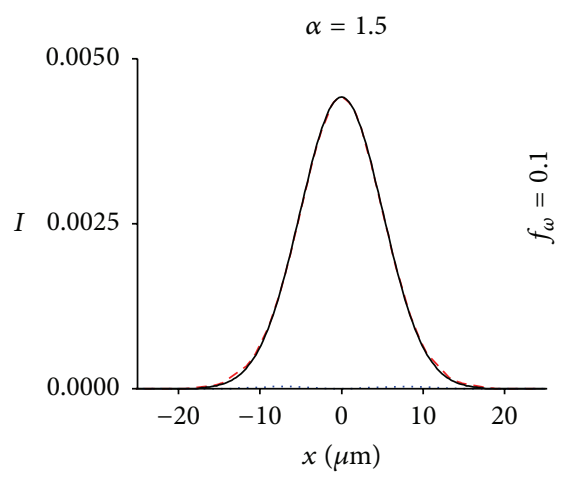

(a3)

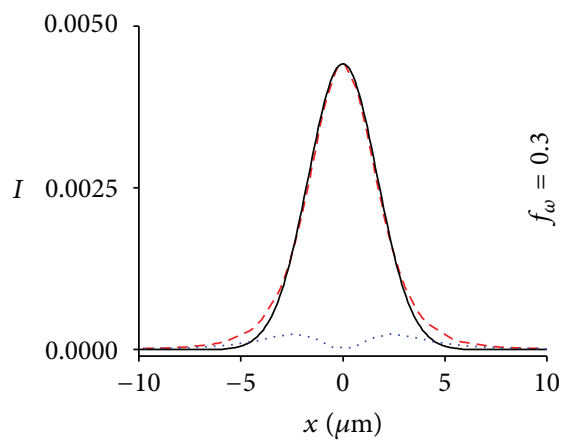

(b3)

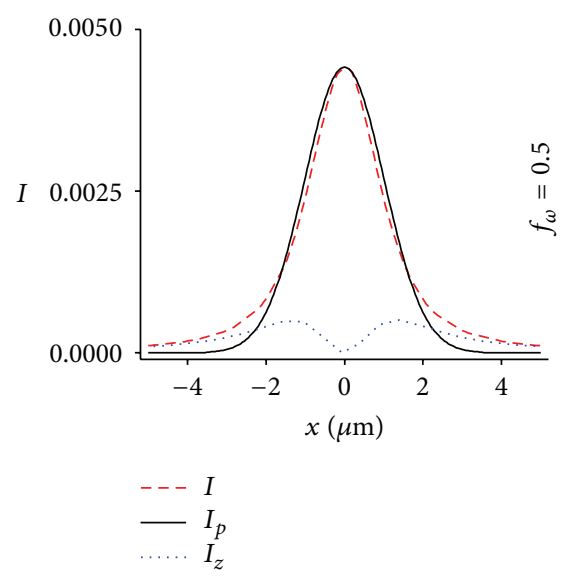

(c3)

FiguRE 3: Intensity distributions $I(x, y, z), I_{z}(x, y, z)$, and $I_{p}(x, y, z)$ of elliptical Gaussian beam in the plane $z=10 z_{r}$ for different parameters $f_{\omega}$ and elliptic coefficient $\alpha$ : (a1) $f_{\omega}=0.1, \alpha=0.8$; (b1) $f_{\omega}=0.3, \alpha=0.8$; (c1) $f_{\omega}=0.5, \alpha=0.8$; (a2) $f_{\omega}=0.1, \alpha=1$; (b2) $f_{\omega}=0.3, \alpha=1$; (c2) $f_{\omega}=0.5, \alpha=1$; (a3) $f_{\omega}=0.1, \alpha=1.5$; (b3) $f_{\omega}=0.3, \alpha=1.5$; (c3) $f_{\omega}=0.5, \alpha=1.5$.

distribution $I$ and corresponding paraxial result $I_{p}$ are almost coincident. While the parameters $f_{\omega}$ are increased to 0.3 (see Figures $3(\mathrm{~b} 1)-3(\mathrm{~b} 3)), I_{z}$ becomes strong, so $I$ and $I_{p}$ began to show slight difference. When $f_{\omega}$ is further increased to 0.5 , $I_{z}$ becomes more strong, and the difference between $I$ and $I_{p}$ increased obviously (see Figures 3(c1)-3(c3)). As a result, no matter what the value of $\alpha$ is, the nonparaxial conclusions of the elliptical Gaussian beam should be considered when $f_{\omega}$ is large. Conversely, the paraxial approximation of elliptical Gaussian beam is valid when $f_{\omega}$ is small. Furthermore, the light peak intensity value will decrease with increasing the elliptic coefficient $\alpha$ in the same observation plane, no matter what value of $f_{\omega}$ is. However, the larger the value of parameters $f_{\omega}$ is, the smaller the spot size of beam is, no matter what value of elliptic coefficient $\alpha$ is.

Figure 4 shows the contour graphs of intensity distributions $I_{x}, I_{z}$, and $I$ of nonparaxial elliptical Gaussian beams for elliptic coefficient $\alpha=1.5$ in the plane $z=10 z_{r}$, and the corresponding paraxial result $I_{p}$ is also given in Figure 4 . The parameter $f_{\omega}$ is chosen as $0.1,0.3$, and 0.5 from the first row to the third row, respectively. As shown in Figure 3, when $f_{\omega}$ is small, the longitudinal component $I_{z}$ is very 


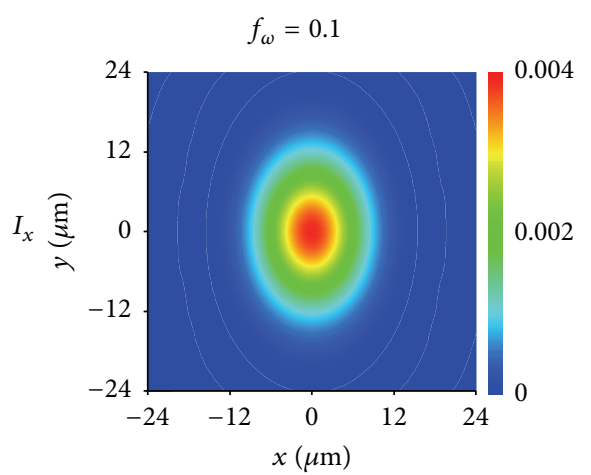

(a1)

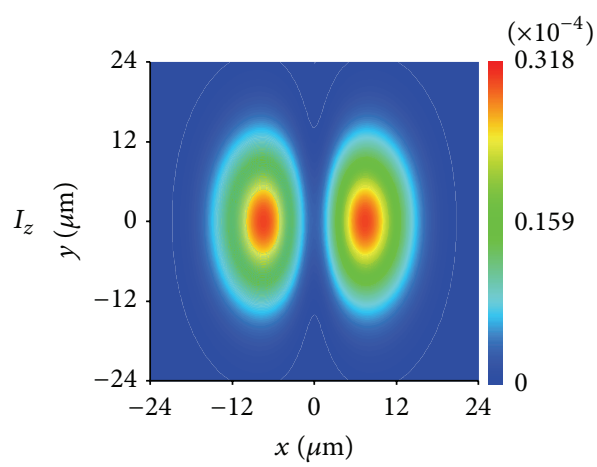

(a2)

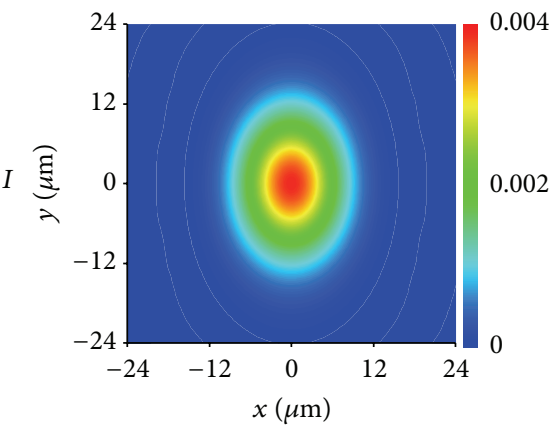

(a3)

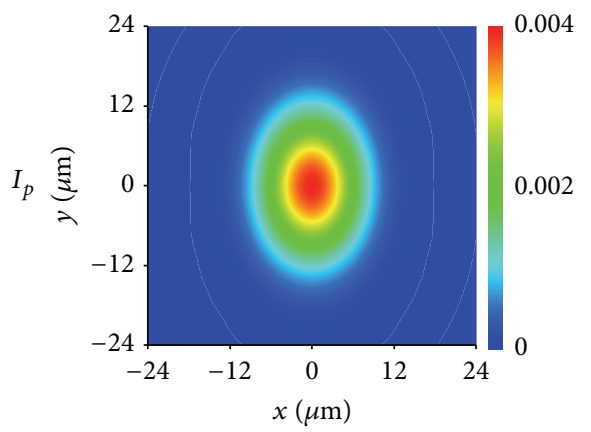

(a4)

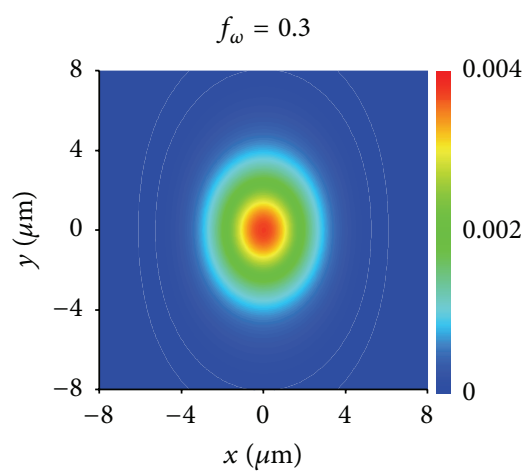

(b1)

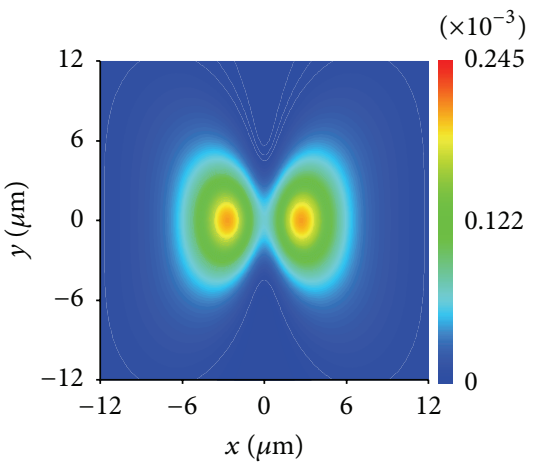

(b2)

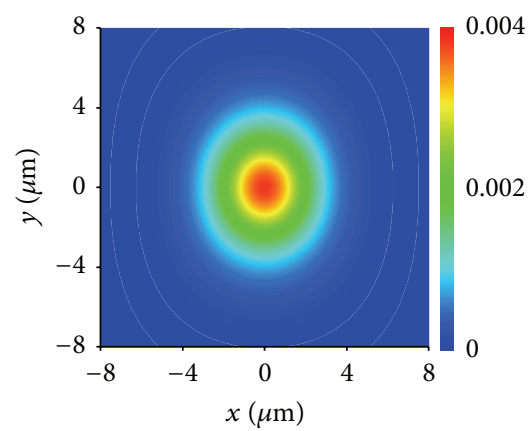

(b3)

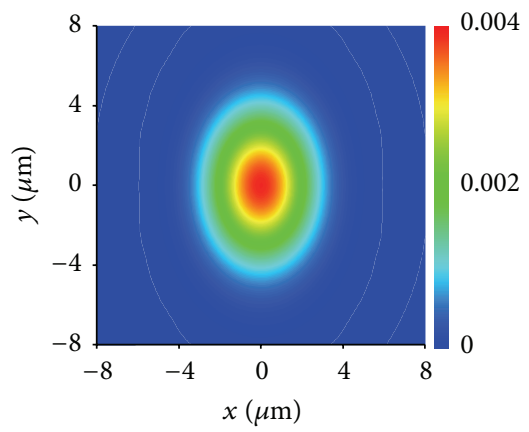

(b4)

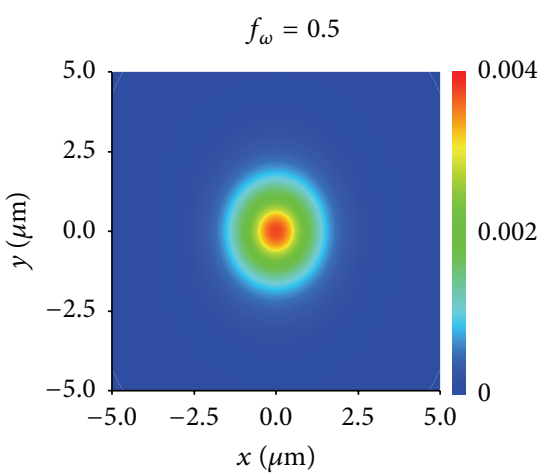

(c1)

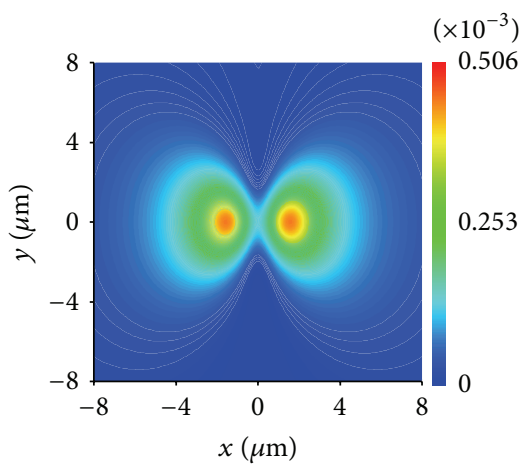

(c2)

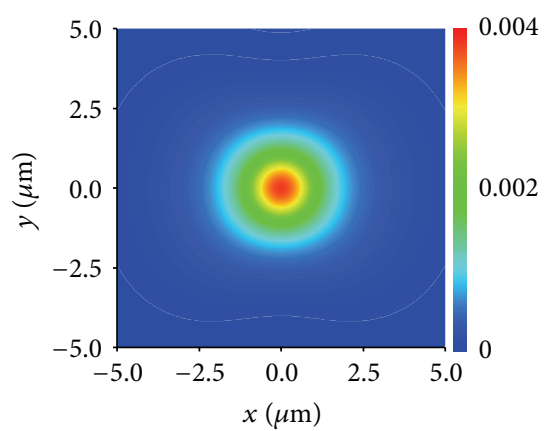

(c3)

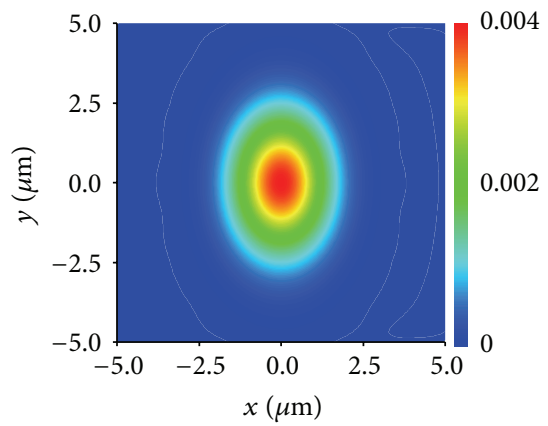

(c4)

Figure 4: Contour graphs of intensity distributions $I_{x}(x, y, z), I_{z}(x, y, z), I(x, y, z)$, and $I_{p}(x, y, z)$ of elliptical Gaussian beam with elliptic coefficient $\alpha=1.5$ in the plane $z=10 z_{r}$ for different parameters $f_{\omega}$ : (a1)-(a4) $f_{\omega}=0.1$; (b1)-(b4) $f_{\omega}=0.3$; (c1) $-(\mathrm{c} 4) f_{\omega}=0.5$.

small and can be neglected; hence the beam profiles of total intensity distribution $I$ and corresponding paraxial result $I_{p}$ are visibly similar. Figures $4(\mathrm{a} 1)-4(\mathrm{a} 4)$ also show that $I_{z}$ can be neglected, and the paraxial approximation is valid when $f_{\omega}$ is small. However, when $f_{\omega}$ is chosen as 0.3 (see Figures 4(b1) $-4(\mathrm{~b} 4)), I_{z}$ becomes strong, and the spots of $I$ and $I_{p}$ show a little distinction. As $f_{\omega}$ is further increased to $0.5, I$ and $I_{p}$ show obvious difference (see Figures $4(\mathrm{cl})-4(\mathrm{c} 4)$ ). In 
other words, the contribution of the longitudinal component $I_{z}$ would become significant, and the nonparaxial conclusions of elliptical Gaussian beam should be adopted when $f_{\omega}$ is large.

\section{Conclusions}

In this paper, based on the vectorial Rayleigh-Sommerfeld diffraction integral formulae, we have derived the analytical expressions for a vectorial elliptical Gaussian beam's nonparaxial propagating in free space, and the paraxial approximation expression has also been examined as a special case. The evolution of the beam's intensity and shape with different elliptic coefficient $\alpha$ and different waist width related parameter $f_{\omega}$ is illustrated by numerical examples. Results show that, with increasing propagation distance $z$, all contours of the transverse cross sections of nonparaxial propagation of the elliptical Gaussian beams preserve Gaussian type when $f_{\omega}$ is small, while all contours of the transverse cross sections of nonparaxial propagation of the elliptical Gaussian beams would change to Gaussian-like type when $f_{\omega}$ is large. Meanwhile, whether parameter $f_{\omega}$ is large or small, the peak intensity value decreased with increasing the propagation distance, and the larger the elliptic coefficient $\alpha$ is, the faster the peak intensity value decreases. In addition, numerical results also show that no matter what value of elliptic coefficient $\alpha$ is, when parameter $f_{\omega}$ is small, the paraxial approximation of elliptical Gaussian beam is effective; when parameter $f_{\omega}$ is large, the nonparaxial conclusions of the elliptical Gaussian beam should be adopted. These characteristics of vectorial elliptical Gaussian beam might find applications in modern optics.

\section{Conflict of Interests}

The authors declare that there is no conflict of interests regarding the publication of this paper.

\section{Acknowledgments}

This work is supported by the National Science Foundation of China (11547002 and 11447235), Jiangxi Provincial Natural Science Foundation of China (20142BAB212003), and China Scholarship Council 201508360027.

\section{References}

[1] E. Kapon, J. Katz, and A. Yariv, "Supermode analysis of phaselocked arrays of semiconductor lasers," Optics Letters, vol. 9, no. 4, pp. 125-127, 1984.

[2] J. Faist, F. Capasso, D. L. Sivco, C. Sirtori, A. L. Hutchinson, and A. Y. Cho, "Quantum cascade laser," Science, vol. 264, no. 5158, pp. 553-556, 1994.

[3] D. A. Fletcher, K. E. Goodson, and G. S. Kino, "Focusing in microlenses close to a wavelength in diameter," Optics Letters, vol. 26, no. 7, pp. 399-401, 2001.

[4] K. J. Vahala, “Optical microcavities," Nature, vol. 424, no. 6950, pp. 839-846, 2003.
[5] R. Borghi, M. Santarsiero, and M. A. Alonso, "Highly focused spirally polarized beams," Journal of the Optical Society of America A: Optics and Image Science, and Vision, vol. 22, no. 7, pp. 1420-1431, 2005.

[6] Z. R. Liu and D. M. Zhao, "Radiation forces acting on a Rayleigh dielectric sphere produced by highly focused elegant Hermitecosine-Gaussian beams," Optics Express, vol. 20, no. 3, pp. 28952904, 2012.

[7] Z. R. Liu and D. M. Zhao, "Optical trapping Rayleigh dielectric spheres with focused anomalous hollow beams," Applied Optics, vol. 52, no. 6, pp. 1310-1316, 2013.

[8] S. Nemoto, "Nonparaxial Gaussian beams," Applied Optics, vol. 29, no. 13, pp. 1940-1946, 1990.

[9] R. K. Luneburg, Mathematical Theory of Optics, University of California Press, Berkeley, Calif, USA, 1966.

[10] M. Lax, W. H. Louisell, and W. B. McKnight, "From Maxwell to paraxial wave optics," Physical Review A, vol. 11, no. 4, pp. 13651370, 1975.

[11] A. Wünsche, "Transition from the paraxial approximation to exact solutions of the wave equation and application to Gaussian beams," Journal of the Optical Society of America B, vol. 9, no. 5, pp. 765-774, 1992.

[12] C. G. Chen, P. T. Konkola, J. Ferrera, R. K. Heilmann, and M. L. Schattenburg, "Analyses of vector Gaussian beam propagation and the validity of paraxial and spherical approximations," Journal of the Optical Society of America A: Optics and Image Science, and Vision, vol. 19, no. 2, pp. 404-412, 2002.

[13] S. R. Seshadri, "Virtual source for a Hermite-Gauss beam," Optics Letters, vol. 28, no. 8, pp. 595-597, 2003.

[14] Z. R. Mei and D. M. Zhao, "Nonparaxial analysis of vectorial Laguerre-Bessel-Gaussian beams," Optics Express, vol. 15, no. 19, pp. 11942-11951, 2007.

[15] D. G. Deng, H. Yu, S. Q. Xu, G. L. Tian, and Z. X. Fan, "Nonparaxial propagation of vectorial hollow Gaussian beams," Journal of the Optical Society of America B: Optical Physics, vol. 25, no. 1, pp. 83-87, 2008.

[16] G. Q. Zhou, "Nonparaxial propagation of a Lorentz-Gauss beam," Journal of the Optical Society of America. B, vol. 26, no. 1, pp. 141-147, 2009.

[17] B. Gu and Y. Cui, "Nonparaxial and paraxial focusing of azimuthal-variant vector beams," Optics Express, vol. 20, no. 16, pp. 17684-17694, 2012.

[18] A. Naqwi and F. Durst, "Focusing of diode laser beams: a simple mathematical model," Applied Optics, vol. 29, no. 12, pp. 1780$1785,1990$.

[19] K. L. Duan and B. D. Lü, "Propagation properties of vectorial elliptical Gaussian beams beyond the paraxial approximation," Optics and Laser Technology, vol. 36, no. 6, pp. 489-496, 2004.

[20] B. D. Lü and K. L. Duan, "Nonparaxial propagation of vectorial Gaussian beams diffracted at a circular aperture," Optics Letters, vol. 28, no. 24, pp. 2440-2442, 2003.

[21] K. L. Duan and B. D. Lü, "Vectorial nonparaxial propagation equation of elliptical Gaussian beams in the presence of a rectangular aperture," Journal of the Optical Society of America $A$, vol. 21, no. 9, pp. 1613-1620, 2004. 

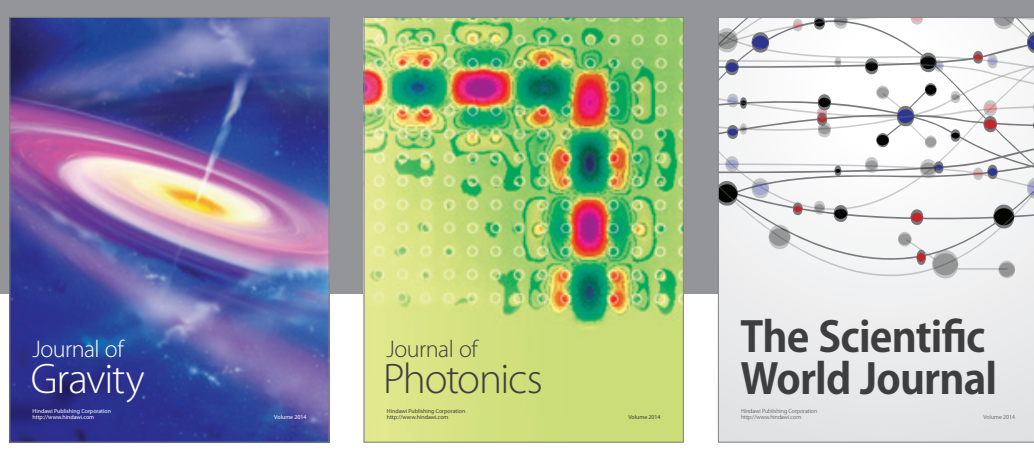

The Scientific World Journal
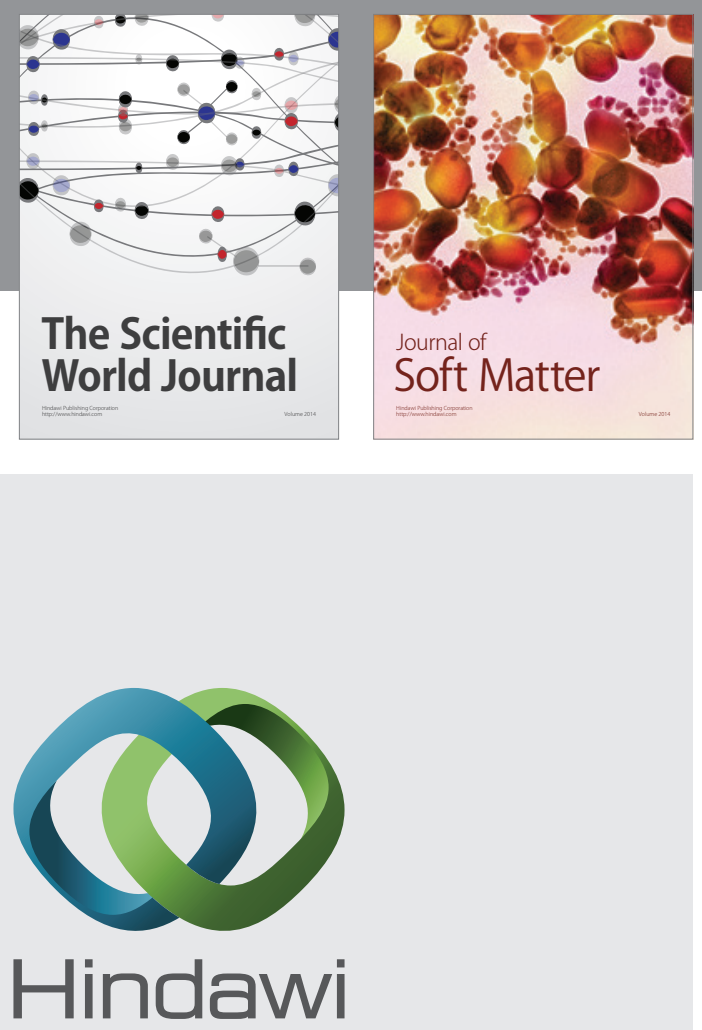

Submit your manuscripts at

http://www.hindawi.com

nternational Journal of

Statistical Mechanics
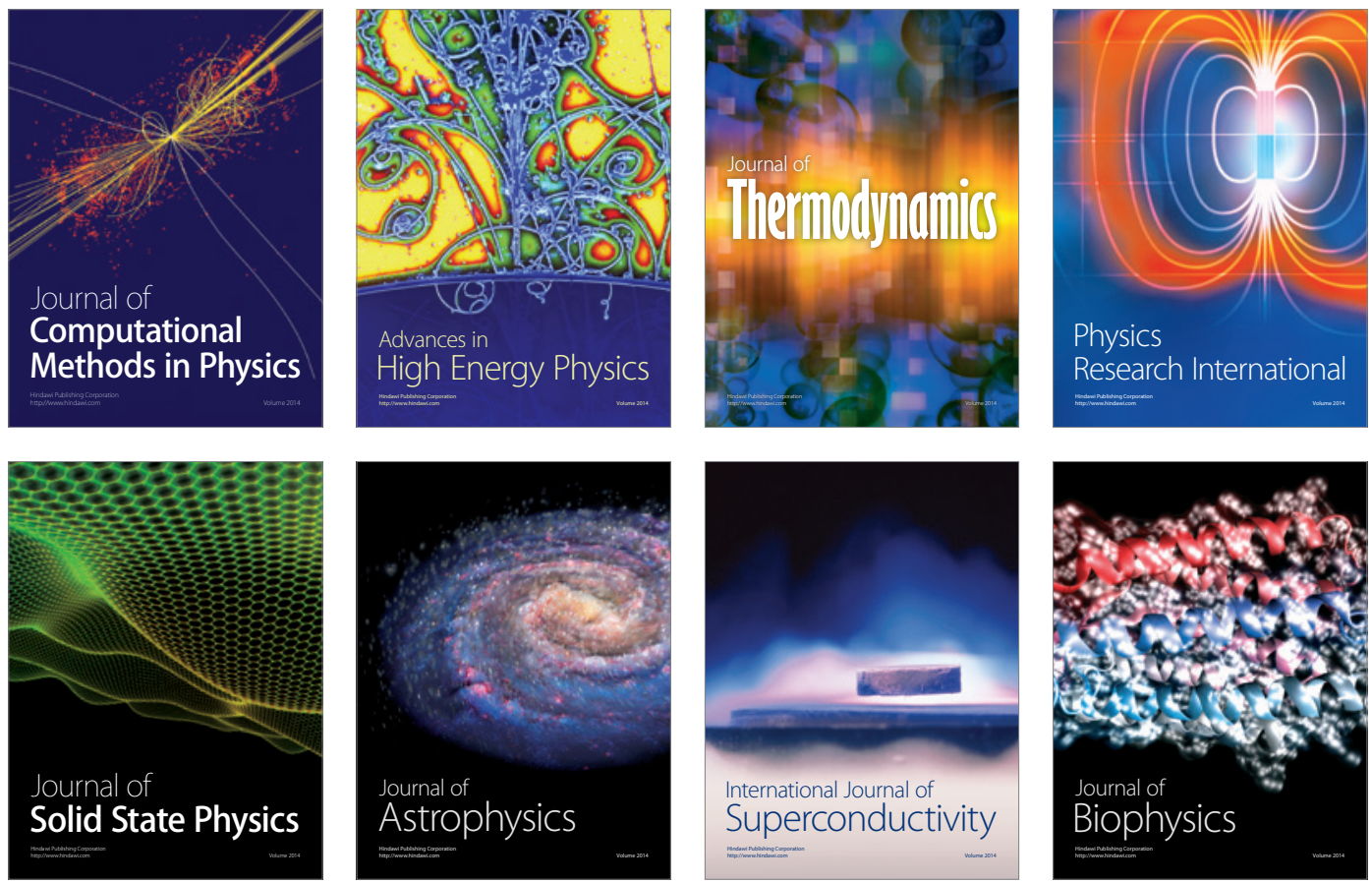
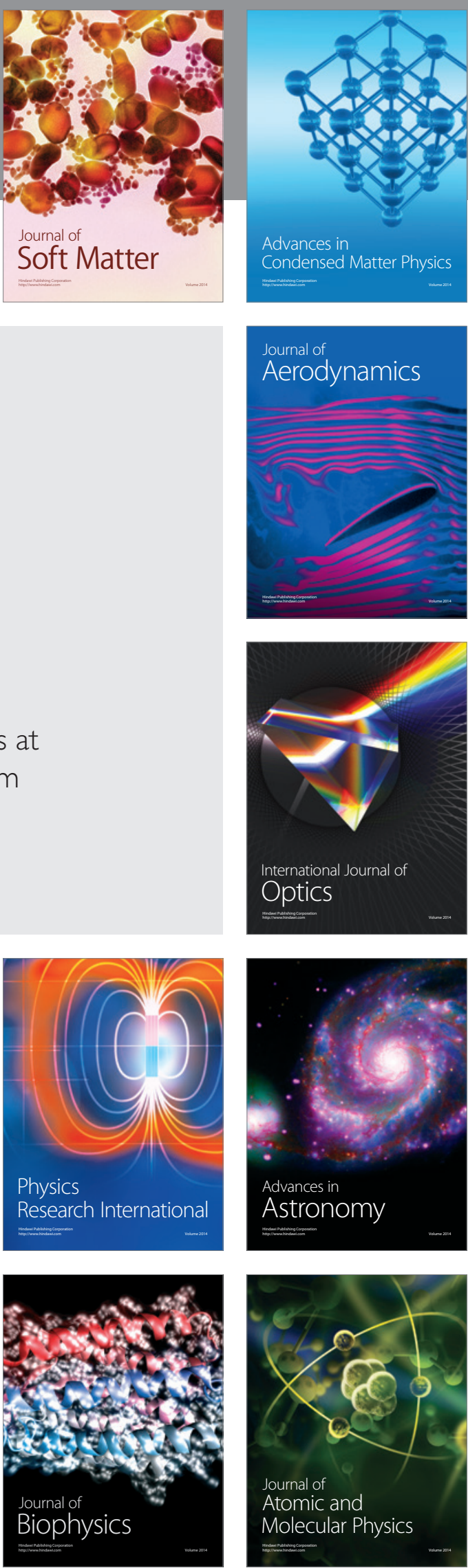